Klage eines Arbeitnehmers, der gegen einen nach dem TzBfG (i.d.F. vom 21. Dezember 2000) befristeten Arbeitsvertrag geklagt hatte. Das BAG gab unter Berufung auf das MangoldUrteil der Klage statt. Honeywell als Arbeitgeber hat Verfassungsbeschwerde gegen die Entscheidung erhoben. Das BVerfG hat nun zu entscheiden, ob das Mangold-Urteil des EuGH die nationalen Gerichte bindet oder ob ein im Sinne der
Rechtsprechung des BVerfG ,ausbrechender Rechtsakt' vorliegt ${ }^{20}$, wie Honeywell vorträgt. Eine sicherlich spannende Entscheidung, die dem BVerfG erneut Gelegenheit geben wird, sein Verhältnis zum EuGH zu klären.

\title{
„Häusliche Gewalt“ - Notwendigkeit und Inhalt einer bundeseinheitlichen Definition für die Justiz
}

\section{Dagmar Freudenberg \\ Vorsitzende der Kommission Strafrecht des djb; Staatsanwältin, MJ - Landespräventionsrat - Niedersachsen, Hannover}

Seit mehr als zehn Jahren wird in der Gesellschaft und in der Politik die Bekämpfung häuslicher Gewalt als notwendig erachtet. Rechtliche, gesellschaftliche und soziale Instrumentarien wurden und werden entwickelt. Verschiedene Professionen wie Polizei und Sozialwissenschaften erarbeiten Interventionsstrategien, teils unter Beteiligung der Justiz. Dabei haben die Beteiligten professionsbezogene Definitionen der häuslichen Gewalt entwickelt, die sie in ihrem Feld jeweils für notwendig und handhabbar halten. Um die bei der Justiz bundesweit anfallenden Verfahren vergleichbar erheben zu können, ist es notwendig, auch hier eine einheitliche Definition des Begriffs „Häusliche Gewalt“ zugrunde zu legen.

\section{Bereits vorhandene Definitionen in verschiedenen Kontexten}

Bundes- oder landesweit ist eine einheitliche Definition des Begriffs bisher nicht vorhanden. Soweit mit dem Begriff in den verschiedenen professionellen Zusammenhängen gearbeitet wird, erfolgt nach einer Umfrage bei den in den Bundesländern bestehenden Landeskoordinierungsstellen und -projekten gegen häusliche Gewalt die Abgrenzung nach professions- bzw. phänomen-spezifischen Gesichtspunkten.

Im Bereich der Polizei werden die Sachverhalte, soweit anhand der mitgeteilten Definitionen erkennbar, anhand der bestehenden oder ehemaligen Beziehung zwischen den Partnern in Fällen von physischer, psychischer oder sexueller Gewalt von anderen Fallkonstellationen abgegrenzt.

Für den Bereich der Polizei in Niedersachsen ist folgende Definition festgelegt:

Häusliche Gewalt umfasst alle Erscheinungsformen der physischen, sexuellen und/oder psychischen Gewalt zwischen Menschen, die in nahen Beziehungen stehen oder standen. ${ }^{1}$

Soweit im Bereich der Staatsanwaltschaften in den Bundesländern eine Definition vorliegt, wird die Abgrenzung teilweise anhand von Deliktskatalogen vorgenommen.

In Niedersachsen wurde bisher auf der Basis der Erarbeitung durch den Praxisbeirat der Landeskoordinierungsstelle häus- liche Gewalt beim Landespräventionsrat im Bereich der bereits existierenden Sonderdezernate in der Justiz zugrunde gelegt, dass sich unter dem Oberbegriff der Gewalt im sozialen Nahraum Gewalt in Partnerschaften oder Ex-Partnerschaften (häusliche Gewalt im engeren Sinn), Kindesmisshandlung und sonstige Gewalt im sozialen Nahraum (z.B. Elternmisshandlung, Gewalt gegen Senioren) zusammenfassen lässt. Dabei kommen Nachstellung („Stalking“) und sexuelle Gewalt in allen diesen Bereichen als eine spezielle Form der Gewalt vor.

Häusliche Gewalt im engeren Sinn ist dann die Verletzung der physischen und/oder psychischen Integrität des Opfers unter hetero- oder homosexuellen Paaren in ehemaligen, in Auflösung befindlichen oder bestehenden partnerschaftlichen Beziehungen, wenn die Gewalt unabhängig vom Tatort in Zusammenhang mit der bestehenden oder aufgelösten Beziehung geschieht, unabhängig davon, ob die Beteiligten je zusammengelebt haben oder zusammenleben.

\section{Kriterien zur Abgrenzung des Begriffs}

Bei der Abgrenzung der häuslichen Gewalt von anderen Erscheinungsformen der Kriminalität sind verschiedene Kriterien zu berücksichtigen.

\section{Betroffener Personenkreis/Beteiligte}

Zur Abgrenzung ist zunächst der Kreis der als Täter und Opfer betroffenen Personen zu bestimmen, um einer Vermischung von Verfahren entgegenzuwirken. Dies hängt auch mit der Notwendigkeit der Bearbeitung derartiger Verfahren durch besonders geschulte Dezernent(inn)en in der Staatsanwaltschaft zusammen. Besonderheiten in der Bearbeitung von Verfahren wegen häuslicher Gewalt ergeben sich aus dem Umstand, dass bei Straftaten gegen Personen, die dem (der) Täter(in) nahestehen, in der weit überwiegenden Zahl der

1 Runderlass des Niedersächsischen Ministeriums für Inneres und Sport vom 17.12.2007-im Einvernehmen mit dem Niedersächsischen Ministerium für Soziales, Frauen, Familie und Gesundheit und dem Niedersächsischen Justizministerium - in der Handreichung für die Polizei II „Gewalt gegen Frauen im häuslichen Bereich“, 6. 
Fälle ein Rollenkonflikt auf der Seite des (der) Verletzten aus der Opfereigenschaft - der Verpflichtung, als Zeuge (Zeugin) sachlich zur Aufklärung beitragen zu müssen - und der Beziehungsebene zum (zur) Täter(in) entsteht. Dieser Rollenkonflikt verursacht - neben weiteren beziehungsbezogenen Besonderheiten - die Ambivalenz der (der) Verletzten und macht sie zugleich für den (die) Täter(in) erpressbar. Das Funktionieren des Ermittlungs- und Strafverfahrens bei der Strafverfolgung auf der Basis des staatlichen Gewaltmonopols zur Identifizierung, Ächtung und Ahndung von Straftaten wird durch diesen Rollenkonflikt und die sich daraus ergebenden Konsequenzen entscheidend beeinflusst. Wesentliche Ursache für den Rollenkonflikt ist dabei die - häufig ungeklärte - Beziehung zwischen Täter(in) und Opfer, die bei der Bearbeitung derartiger Verfahren nicht nur in Zusammenhang mit Fällen des $\mathbb{S} 52$ StPO, sondern insgesamt die Ermittlung und Feststellung des tatsächlichen Geschehensablaufs durch intensivierten Aufklärungseinsatz auch seitens Staatsanwaltschaften und Gerichte erforderlich macht, um den staatlichen Strafanspruch durchzusetzen. Als wesentliches Kriterium zur Abgrenzung der Fälle häuslicher Gewalt ist deshalb die bestehende oder ehemalige Beziehung zwischen Täter(in) und Opfer festzulegen.

Dabei ist das Geschlecht der als Täter und Opfer beteiligten Personen nicht von Bedeutung. Art. 3 GG gebietet die Gleichbehandlung homosexueller, heterosexueller und lesbischer Beziehungen, so dass alle diese Beziehungsarten einzubeziehen sind.

Bei der Abgrenzung der Fälle häuslicher Gewalt ist indessen noch eine weitere Besonderheit auf der Opferseite zu beachten: Verletzt werden durch die Gewalthandlungen nicht nur die unmittelbar durch die Handlungen getroffenen Partner(innen). Nach neueren anerkannten wissenschaftlichen Erkenntnissen erleiden auch die in die Beziehung von Täter(in) und Opfer einbezogenen Kinder häufig akute und längerfristige schwerwiegende, zumeist psychische (Entwicklungs-)Beeinträchtigungen. Dabei werden die minderjährigen Kinder auch nach Beendigung der Gewaltbeziehung und Trennung der Partner mit dem Erlebten insbesondere in Zusammenhang mit Fragen des Sorge- und Umgangsrecht zumeist über lange Zeiträume konfrontiert. Längsschnittuntersuchungen zeigen, dass Gewalterfahrungen in der Kindheit ein hohes Risiko bergen, auch im weiteren Lebensverlauf mit Gewalt konfrontiert zu werden, und dass es einen Zusammenhang zwischen dem (Mit-)Erleben elterlicher Gewalt und dem Erdulden und Ausüben von Gewalt im späteren Leben gibt. Insofern kann Gewalt auch über Generationen fortwirken.

\section{Zeitliche und örtliche Begrenzungen}

Nicht allein dieser Gesichtspunkt gebietet ferner auch eine Einbeziehung von Fallkonstellationen, in denen die Beziehungspartner entweder gar nicht zusammengelebt haben, in Trennung leben oder die Beziehung bereits beendet ist. Die bereits vorliegenden Erkenntnisse wissenschaftlicher Forschung zum Phänomen des Stalking belegen, dass sich mehr als 60 Prozent der Stalkingfälle in Zusammenhang mit häuslicher
Gewalt ereignen, wobei die - akute - Trennungsphase ein um ein Vielfaches erhöhtes Eskalationsrisiko bis hin zur Partnertötung birgt. Allerdings sind verlässliche Feststellungen zur Dauer dieser High-Risk-Phasen nicht möglich. Die praktischen Erfahrungen in Behörden mit bereits bestehendem Sonderdezernat zeigen, dass bei mehrere Jahre überdauerndem Stalkingverhalten mit gleichzeitigen psychischen Auffälligkeiten auf der Täterseite das Risiko für die (den) ehemalige(n) Partner(in) sich nicht prognostizieren lässt, gleichzeitig aber das Risiko von häuslicher Gewalt in neuen oder parallel geführten Beziehungen steigt. Auch wenn dies nicht in der Mehrzahl der Fälle auftritt, ist eine zeitliche Begrenzung der in die Definition einzubeziehenden Beziehungsfälle, wie sie zum Beispiel in Hessen mit einer Zwei-Jahresfrist praktiziert wird, unter präventiven und deeskalierenden Gesichtspunkten nicht zu vertreten.

Wegen der Möglichkeiten der modernen (Tele-)Kommunikation verbietet sich auch eine wie auch immer ausgestaltete regionale Begrenzung in der Definition. Nachstellungshandlungen in (ehemaligen) Beziehungen sind weder durch die Grenzen der Länder noch der Kontinente begrenzt. Bei den in derartig grenzüberschreitenden Fallkonstellationen erforderlichen umfangreichen und zeitaufwendigen Ermittlungen, die zudem einen deutlich erhöhten Ermittlungseinsatz bei den Staatsanwaltschaften erfordern, kann auf das Fachwissen der besonders geschulten Sonderdezernent(inn)en bei den Staatsanwaltschaften nicht verzichtet werden.

\section{Abgrenzung nach der Art der Delikte}

Der Begriff der häuslichen Gewalt impliziert ein Über-Unterordnungsverhältnis, eben „Gewalt“, das definitorisch umgrenzt werden muss. Ohl weist in ihrer Beschreibung häuslicher Gewalt als gesellschaftliches Problem vor dem Hintergrund neuerer Erkenntnisse zu Recht darauf hin, dass die Formen sexueller und psychischer Gewalt in diesem Kontext stärker in den Blick genommen werden müssen, weil „sie die körperlichen Aggressionen begleiten bzw. selbst einen Teil der Angriffe darstellen können und insbesondere im Hinblick auf die gesundheitlichen Folgen nicht unterschätzt werden dürfen." 2 Als Formen anderer, „psychischer“ Gewalt differenziert sie neben der

\section{nörperlichen Gewalt}

- die sexuelle Gewalt,

- die ökonomische Gewalt, bei der durch Einteilung des Haushaltsbudgets und/oder Verhinderung eigener Berufstätigkeit finanzielle Abhängigkeit der Partnerin erzeugt und diese dadurch der Willkür und Gunst des Täters ausgeliefert wird,

- die psychische Gewalt, bei der die Partnerin angeschrien, gedemütigt, beleidigt, bedroht, herabgesetzt und/oder durch Sachbeschädigungen eingeschüchtert wird,

2 Dagmar Ohl, Häusliche Gewalt - Beschreibung eines gesellschaftlichen Problems, FPR 2005, 6 ff. 
- die emotionale Gewalt, bestehend vor allem in der Kontrolle beinahe aller Lebensbereiche der Partnerin, ihrer emotionalen Zurückweisung und der vollständigen Verweigerung einer Anerkennung ihrer Person, und

- die soziale Gewalt, bei der durch Einwirkung auf das soziale Umfeld der Partnerin, also eigene oder gemeinsame Kinder oder andere Angehörige, durch den Täter Druck erzeugt und die Entscheidungs- und Verfügungsmacht über alle Familienmitglieder beansprucht wird und/oder beinahe alle Lebensäußerungen der Partnerin auch in der Öffentlichkeit abgewertet werden und sie so verunsichert und im Freundes- und Bekanntenkreis bloßgestellt wird.

Im Strafrecht wird die Gewalt unterschieden in die vis absoluta und die vis compulsiva, die willensbeugende Gewalt.

Fälle der physischen Gewalt, also der vis absoluta, sind in den vorhandenen Straftatbeständen wie Körperverletzung, sexuelle Nötigung und Vergewaltigung, versuchte Tötungsdelikte etc. zumeist trennscharf umschrieben und erfasst und gehören ohne Ausnahme in den Bereich der häuslichen Gewalt.

Schwieriger zu entscheiden ist dies für den Bereich der psychischen Gewalt. Die von Ohl in ihrer Differenzierung beschriebenen Formen der psychischen, ökonomischen, emotionalen und sozialen Gewalt sind nur bedingt von den Tatbeständen der Körperverletzung, der Bedrohung und der Nötigung, der Nachstellung, der Beleidigung, der Verleumdung und üblen Nachrede oder dem Recht am eigenen Bild erfasst. Unter dem Gesichtspunkt der einschränkenden Auslegung der Nötigung, wie sie vom BVerfG vorgegeben wurde, ist eine extensive Ausweitung der Strafbarkeit derartiger Verhaltensweisen dem Gesetzgeber vorbehalten. Gleichwohl können diese typischerweise in Fällen häuslicher Gewalt auftretenden Verhaltensweisen in bestimmten Fallkonstellationen unter die vorgenannten Straftatbestände zu subsumieren sein. Das bedeutet, dass alle diese Straftatbestände auch in den Zuständigkeitsbereich der Definition gehören.

Die in einigen Bundesländern bisher praktizierte Eingrenzung häuslicher Gewalt auf bestimmte Straftatbestände wie Körperverletzung, Freiheitsberaubung, Nötigung, Bedrohung, Nachstellung (Stalking) und Vergehen gegen $\mathbb{S} 4 \mathrm{Gew}-$ SchG, teilweise auch Sachbeschädigung, Raub, Erpressung, Misshandlung von Schutzbefohlenen und Verletzung der Fürsorge- und Erziehungspflicht unter teilweisem Ausschluss von Beleidigung, Hausfriedensbruch und Vermögensdelikten belegt die Uneinheitlichkeit der Sicht auf das Phänomen der häuslichen Gewalt und erscheint als Begrenzung der Definition weder schlüssig noch geeignet. Im Zusammenhang mit dem Phänomen der häuslichen Gewalt sind die möglichen Erscheinungsformen und Tathandlungen - ähnlich wie im Zusammenhang mit dem Straftatbestand der Nachstellung so vielfältig wie das menschliche Handeln im Allgemeinen auch. So ergeben sich in der Praxis nicht selten auch Delikte wie gefährlicher Eingriff in den Straßenverkehr, versuchte Brandstiftung, (versuchtes) Herbeiführen einer Explosions- gefahr, aber auch Verletzung der Unterhaltspflicht und Vergehen gegen $\mathbb{} 17$ TierSchG. Eine Ausgrenzung einzelner Delikte wie insbesondere auch der Sexualdelikte kann zu divergierenden Beurteilungen bei der Einschätzung der Glaubhaftigkeit der Angaben der (des) Verletzten und - dem folgend - im Verfahrensabschluss zu abweichenden Einschätzungen und Entscheidungen führen, die die Verfahrensführung erheblich beeinträchtigen können und den Verfahrensbeteiligten letztlich nicht zu vermitteln sind. Dies gilt auch im Zusammenhang mit der Rechtskraftwirkung von Entscheidungen und dem Grundsatz ne bis in idem.

Eine Eingrenzung der Definition über die Straftatbestände kommt folglich nicht in Betracht.

\section{Differenzierung zwischen amtsanwaltlichem und staats- anwaltlichem Dezernat}

Die Unterscheidung nach amts- und staatsanwaltschaftlichem Dezernat ergibt sich aus den in den Bundesländern geltenden, teils divergierenden Anordnungen über Organisation und Dienstbetrieb in den Staatsanwaltschaften (OrgStA). Für die Frage der Definition häuslicher Gewalt spielt sie im Ergebnis keine Rolle. Die Verteilung kann unabhängig von der Definition behördenintern unter dem Gesichtspunkt des gesamten Fallaufkommens im Zuständigkeitsbereich der Staatsanwaltschaft in Zusammenhang mit dem Sonderdezernat entschieden werden. Dabei kann durchaus die häufig nach Deliktsschwere vorgenommene Differenzierung zugrunde gelegt werden. Wegen der regelmäßig hohen Fallbelastung im amtsanwaltschaftlichen Dezernat erscheint die Übertragung in Fällen des Stalking gem. $\$ 238$ Absatz 1 StGB, die stets auf eine potentielle Eskalationsgefahr hin zu prüfen sind, und die Fälle der (versuchten) gefährlichen Körperverletzung gem. $\int 224$ Abs. 1 Nr. 2 und Nr. 5 StGB, die zumeist die Abgrenzungsproblematik zum versuchten Tötungsdelikt mit besonderer gerichtlicher Zuständigkeit enthalten, problematisch.

Diese Grundüberlegung gilt auch in allen Fällen, in denen Kinder und Jugendliche direkt oder indirekt als zur Familie gehörig mit betroffen sind. Unter dem Gesichtspunkt des Jugendschutzes (Nr. 31, 35 MiStra) bietet sich insoweit eher eine Bearbeitung im Staatsanwaltsdezernat an.

\section{Definition}

Auf der Basis vorgenannten Überlegungen ergibt sich folgende Definition „Häusliche Gewalt“:

Häusliche Gewalt ist - kumulativ oder alternativ - die Verletzung der physischen oder psychischen Integrität des Opfers in ehemaligen, in Auflösung befindlichen oder bestehenden partnerschaftlichen Beziehungen bei heterooder homosexuellen Paaren, wenn die Tat-unabhängig vom Tatort - in Zusammenhang mit der gewünschten, bestehenden oder aufgelösten Beziehung geschieht, unabhängig davon, ob die Beteiligten je zusammengelebt haben oder zusammenleben. Auf die Art der in Frage kommenden Delikte kommt es bei der Einordnung nicht an. 


\section{Statistische Erfassung}

Eine Erfassung der Verfahren häuslicher Gewalt bei der Staatsanwaltschaft kann, wenn sie in gesonderten Sachgruppen erfolgt, die Auswertung der Verfahren auch für politische und wissenschaftliche Zwecke erleichtern. Soweit bisher überhaupt eine statistische Erfassung erfolgt, geschieht dies auf der Basis der in einzelnen Bundesländern ${ }^{3}$ unterschiedlich vorgegebenen Definitionen lediglich für die Staatsanwaltschaften. Die Einführung gesonderter Sachgruppen in der Statistik für die Staatsanwaltschaften und Gerichte auf der Basis der oben genannten Definition würde eine Prüfung des Verfahrensausgangs bei den Staatsanwaltschaften ebenso wie bei den Ge- richten in erster Instanz ermöglichen, da die Sachgruppen insoweit einheitlich verwendet werden. Der Verfahrensverlauf und damit zumindest teilweise die Effektivität der Bekämpfung häuslicher Gewalt durch die Justiz würden dadurch im Längsschnitt überprüfbar. Weitere und neue Instrumente könnten auf dieser Basis entwickelt und evaluiert, ineffektive Maßnahmen im Bereich der Justiz reformiert werden.

3 Hessen, Mecklenburg-Vorpommern, Schleswig-Holstein; in Niedersachsen ist eine flächendeckende Erfassung parallel zu den zum 1.7.2010 flächendeckend einzurichtenden Sonderdezernaten der Staatsanwaltschaften vorgesehen.

\section{Das Sorgerecht der nichtehelichen Väter}

\section{Dr. Angelika Nake \\ Vorsitzende der Kommission Zivil-, Familien- und Erbrecht des djb; Rechtsanwältin, Darmstadt}

Mit Urteil vom 3. Dezember 2009 hat der Europäische Gerichtshof für Menschenrechte (EGMR) zu Art. 8 I, Art. 14 EMRK und $\$ 1626$ a II BGB entschieden, dass Deutschland die Väter nichtehelicher Kinder beim Zugang zur gemeinsamen Sorge diskriminiere. Das Gericht weist insoweit darauf hin, dass es keine objektive Rechtfertigung dafür gibt, dass das Sorgerecht des nichtehelichen Vaters einer gerichtlichen Einzelfallprüfung nicht zugänglich ist. ${ }^{1}$

Dem lag folgender Fall zugrunde: Der 1964 geborene Beschwerdeführer ist Vater eines außerhalb einer Ehe im Jahre 1995 geborenen Kindes. Die Eltern lebten seit 1993 zusammen und trennten sich im August 1998. Bis Januar 2001 wohnte das Kind bei dem Vater, die Mutter wohnte in einer anderen Wohnung, allerdings im gleichen Haus. Im Januar 2001 zog die Mutter dann mit dem Kind aus. Der Vater hatte mit dem Kind einen umfangreichen Umgang, er sah das Kind Mittwochnachmittag bis Donnerstagmorgen und Sonntag ab 10 Uhr bis Montagmorgen sowie die hälftigen Ferien. Insgesamt etwa vier Monate im Jahr. Die Mutter wollte die Sorgeerklärung nicht abgeben, so dass der Vater im Jahr 2001 die gemeinsame Sorge beim Familiengericht beantragte. Im Instanzenzug wurde der Antrag abgelehnt bzw. zur Verfassungsbeschwerde nicht angenommen.

Der Europäische Gerichtshof führt zur Verletzung des Art. 14 i.V.m. Art. 8 EMRK aus, dass eine Rechtfertigung für die Ungleichbehandlung nicht bestehe, anders als das BVerfG in seiner Grundsatzentscheidung vom 29. Januar 2003. ${ }^{2}$ Hier hat das BVerfG festgestellt, dass das Kindeswohl es verlange, dass das Kind ab seiner Geburt eine Person hat, die für es rechtsverbindlich handeln kann. Angesichts der Unterschiedlichkeit der Lebensverhältnisse, in die nichteheliche Kinder hineingeboren werden, sei es verfassungsgemäß, dass die Mut- ter die elterliche Sorge alleine habe. Die Mutter kann dann im Rahmen einer Sorgeerklärung die Mitsorge auf den Vater übertragen. Zum Schutz des Kindeswohls sei es aber gerechtfertigt, dass zunächst die Mutter das Sorgerecht erhält. Das Gericht akzeptierte auch, dass es gewichtige Gründe dafür geben kann, das Sorgerecht nicht jedem nichtehelichen Vater zu übertragen, zum Beispiel dann, wenn Streit oder mangelnde Kommunikation zwischen den Eltern dem Kindeswohl schaden würden.

Im vorliegenden Fall teilt der EGMR diese Bedenken nicht. Er führt aus, dass hier die Eltern gemeinsam gelebt haben, dreieinhalb Jahre in nichtehelicher Lebensgemeinschaft und weitere zwei Jahre noch nachher im gleichen Haus. Das Gericht weist darauf hin, dass dem Vater die Möglichkeit eingeräumt werden muss, gerichtlich überprüfen zu lassen, ob das gemeinsame Sorgerecht dem Kindeswohl dienlich ist. Es stellt anhand der Untersuchung der Bundesregierung fest, dass es nicht nur kindeswohlbezogene Erwägungen der Mütter wären, dem Vater das Mitsorgerecht nicht per Sorgeerklärung übertragen zu lassen. Keine Kindeswohlgefährdung sieht das Gericht in der seiner Ansicht nach notwendigen Klage zur Sorgerechtsübertragung, da diese auch im Rahmen von ehelichen Kindschaftsverhältnissen notwendig sein könne.

Dies ist soweit richtig; auch bei einer Scheidung oder Trennung kommen Anträge zur Übertragung der alleinigen Sorge auf den einen oder anderen Ehegatten vor. Das Sorgerecht wird in den Fällen auf ein Elternteil übertragen, in welchen die Weiterführung der gemeinsamen Sorge kindeswohlwidrig wäre. Das BVerfG legt als Prämisse zugrunde, dass die Ausübung der elterlichen Sorge im Sinne des Kindeswohls ein Mindestmaß an Einverständnis zwischen den Elternteilen voraussetzt. Wenn dieses Mindestmaß an Einverständnis nicht vorliegt, kann ein gemeinsames Sorgerecht kindeswohlför-

1 EGMR, FamRZ 2010, $103 \mathrm{ff}$.

2 NJW 2003, 955; FPR 2003, 205 = FamRZ 2003, 285 